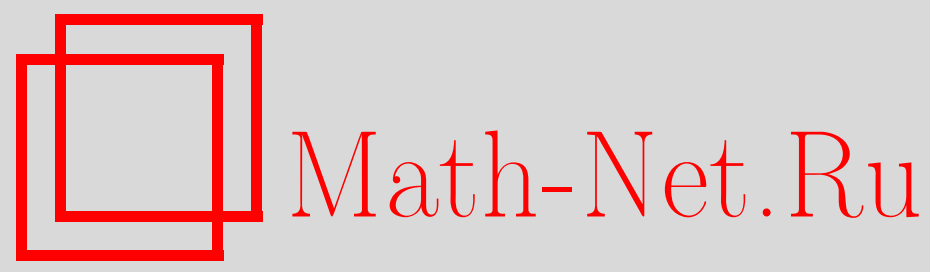

C. В. Чиспияков, О c-формациях с нильпотентными предмаксимальными с-подформациями, Матем. заметки, 2001, том 69, выпуск 1, 113-121

DOI: https://doi.org/10.4213/mzm488

Использование Общероссийского математического портала Math-Net.Ru подразумевает, что вы прочитали и согласны с пользовательским соглашением http://www.mathnet.ru/rus/agreement

Параметры загрузки:

IP : 54.147 .182 .235

26 апреля 2023 г., 18:07:28

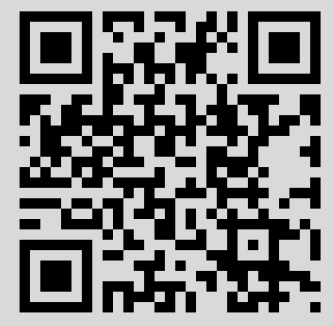




\section{О $c$-ФОРМАЦИЯХ С НИЛЬПОТЕНТНЫМИ ПРЕДМАКСИМАЛЬНЫМИ $c$-ПОДФОРМАЦИЯМИ}

\section{С. В. Чиспияков}

Рассматривается строение композиционных формаций, все предмаксимальные композиционные подформации которых нильпотентны.

Библиография: 14 названий.

Множество групп, содержашее вместе с каждой своей группой $G$ и все групшы, изоморфные $G$, называется $к л а с c о м$. В теории групп понятие класса имеет большое значение. Однако, как самостоятельное направление в рамках теории груп, теория классов начала свое развитие лишь в 30-е годы после опубликования работ Г. Биркгофа [1] и Б.X. Неймана [2]. С выходом в 1963 году работы В. Гашюца [3] началось интенсивное изучение различных классов конечных груп, ключевую роль среди которых заняли формации групा. Таким образом, в рамках теории групп возникло и вполне оформилось новое научное направление - теория формаций.

Формаиия конечных групп $\mathfrak{F}$ называется (разрешимо) насыщенной, если ей принадлежит каждая конечная группа $G$, обладающая такой (разрешимой) нормальной подгруппой $N$, что $G / \Phi(G) \in \mathfrak{F}$. Непустые насыщенные формации называются локальными, а непустые разрешимо насьщенные формации называют композииионными. Насьщенные формации играют значительную роль в теории конечных разрешимых групп. При изучении групп необязательно разрешимых более полезны разрешимо насьшенные формации. Теория локальных формаций в настоящее время является весьма развитым учением, обогашенньм большим числом глубоких теорем и содержательных примеров. Так, в работе [4] были описаны минимальные ненильпотентные локальные формации. Позднее этот результат был развит в работе [5], где были описаны локальные формации, в уравнениях для которых все предмаксимальные локальные подформации нильпотентны.

Теория разрешимо насьщенных формаций развита сравнительно мало, поэтому при изучении произвольных конечных групп на передний план выходят композиционные формации, которые определяются заданием значений экрана на некотором множестве простых групп. Понятие композиционной формации было введено Л. А. Шеметковым в 1973 году на 12-м Всесоюзном алгебраическом коллоквиуме в Свердловске [6]. Одним из подходов к изучению формаций является изучение формаций, у которых некоторая выделенная система подформаций удовлетворяет определенным требованиям, в связи с чем

Работа выполнена при финансовой поддержке Российского фонда фундаментальных исследований, грант № 97-01-00228. 
были разработаны особые методы изучения формаций, связанные с изучением критических формаций.

В данной работе исследуется строение композиционных формаций, у которых все предмаксимальные композиционные подформации нильпотентны. Все рассматриваемые групшы - конечные.

Все необходимые обозначения можно найти в монографии Л. А. Шеметкова [7] или в работе А. Н. Скибы [4]. Приведем некоторые из них.

ОПРЕДЕЛЕНИЕ 1. Экран - отображение $f$ класса $\mathfrak{G}$ во множество всех формаций групп, удовлетворяющих следующим условиям:

1) $f(1)=\mathfrak{G}$

2) $f(G) \subseteq f\left(G^{\varphi}\right) \cap f(\operatorname{Ker} \varphi)$ для любой группы $G$ и любого гомоморфизма $\varphi$ группы $G$.

ОПРЕДЕЛЕНИЕ 2. $K(G)$ - класс всех простых групп, изоморфных композиционньм факторам групшы $G$.

ОПРЕДЕЛЕНИЕ 3. Композииионный экран - это такой экран $f$, что для любой неединичной групшы $G f(G)=\cap f(A)$, где $A$ пробегает $K(G)$.

Напомним, что формации всех абелевых, нильпотентных, разрешимых и простых групп обозначаются $\mathfrak{A}, \mathfrak{N}, \mathfrak{S}, \mathfrak{I}$ соответственно.

ОПРЕДЕЛЕНИЕ 4. Композииионная формация - формация, обладающая по крайней мере одним композиционным экраном.

ОПРЕДЕЛЕНИЕ 5. $\mathfrak{N}_{c}$-критическая формация - такая ненильпотентная композиционная формация, все собственные композиционные подформации которой нильпотентны.

ОПРЕДЕЛЕНИЕ 6 . Группы $A$ и $B$ называются $f$-иниидентными, если либо $A \in$ form $B$, либо $B \in$ form $A$.

ОПРЕДЕЛЕНИЕ 7. Группу $G$ назовем әлементарной $A$-әруппой, если $G=A_{1} \times A_{2} \times$ $\cdots \times A_{n}$, где $A_{i} \cong A, i=1, \ldots, n$.

Для компактного изложения материала примем следующие сокращения: $c$-формаuия - композиционная формация; с-экран - композиционньй экран.

ОПРЕДЕЛЕНИЕ 8. Формация (с-формация) $\mathfrak{H}$ называется максимальной подформаиией (c-подформачией) формации (c-формации) $\mathfrak{F}$, если для каждой формации ( $c$-формации) $\mathfrak{L}$ из $\mathfrak{H} \subseteq \mathfrak{L} \subset \mathfrak{F}$ всегда следует, что $\mathfrak{H}=\mathfrak{L}$.

Лемма 1. Если $G$ - монолитическая группа $c$ монолитом $P$, әде $P$ - элементарная абелева р-группа, $P / 1$ - чентральный главный фактор группы $G u$ $K(G)=K(G / P)$, mo $c$ form $G=c$ form $(G / P)$.

ДокАЗАтЕЛЬСтво. Пусть $\mathfrak{F}=c$ form $G, \mathfrak{H}=c$ form $(G / P)$ и $f, h$ - минимальные $c$-экраны формации $\mathfrak{F}$ и $\mathfrak{H}$ соответственно. Тогда по теореме из [8]

$$
h(A)= \begin{cases}\operatorname{form}\left((G / P) / F_{A}(G / P)\right) & \forall A \in K(G / P), \\ \varnothing & \forall A \in \mathfrak{I} \backslash K(G / P) .\end{cases}
$$


Так как главньй фактор $P / 1 \cong P$ централен в группе $G$, то $P \leq Z(G), P \leq F_{A}(G)$ и, значит, $F_{A}(G / P)=F_{A}(G) / P$ для $A \in \mathfrak{I}$. Тогда по теореме из [8]

$$
f(A)= \begin{cases}\operatorname{form}\left(G / F_{A}(G)\right) & \forall A \in K(G), \\ \varnothing & \forall A \in \mathfrak{I} \backslash K(G) .\end{cases}
$$

Поскольку $K(G)=K(G / P)$, то $f=h$ и по следствию 1 из [8] $\mathfrak{F}=\mathfrak{H}$. Лемма доказана.

ОПреДЕЛЕНИЕ 9. Секиией групшы $G$ назьвается факторгруппа $A / B$, где $A \leq G$, $B G$.

ОПРЕДЕЛЕНИЕ 10. Формационной секцией групшы $G$ называется секция, принадлежащая form $G$.

ОПРЕДЕЛЕНИЕ 11. Группа $G$ называется формачионно критической, если она не принадлежит формации, порожденной всеми ее собственными формационными секциями.

ОПРЕДЕЛЕНИЕ 12. Формационно критическая группа $G$ называется $f$-базисной (cбазисной), если формация form $G(c$ form $G$ ) содержит единственную максимальную подформацию (с-подформацию).

ЛЕмма 2. Если $G$ - монолитическая группа с неабелевым монолитом $P$, то $\mathfrak{H}=c \operatorname{form}(G / P)-$ единственная максимальная $c$-подформация формаиии $\mathfrak{F}=$ $c$ form $G$.

ДокАЗАТЕЛЬСТво. По лемме 3.2.1 из [10] $G$ ная $c$-подформация $\mathfrak{M}$ из $\mathfrak{F}$ имеет внутренний $c$-экран $m$ со следующим строением:

$$
m(A)= \begin{cases}\operatorname{form}(G / P), & A \in K(P), \\ \operatorname{form}\left(G / F_{A}(G)\right) & \forall A \in K(G) \backslash K(P), \\ \varnothing & \forall A \in \mathfrak{J} \backslash K(G)\end{cases}
$$

Пусть $K(P)=(A), b$ - минимальньй $c$-экран формации $\mathfrak{H}=c$ form $(G / P)$. По теореме из $[8] b(D)=\operatorname{form}\left((G / P) / F_{D}(G / P)\right)$ при $D \in K(G / P)$ и $b(D)=\varnothing$ при $D \in \mathfrak{J} \backslash K(G / P)$. Пусть $D \in K(G / P) \backslash(A)$. Тогда в силу леммы 2.8 из [11] $F_{D}(G / P)=F_{D}(G) / P b(D)=$ $m(D)$. Если $A \in K(G / P)$, то $b(A)=\operatorname{form}\left((G / P) / F_{A}(G / P)\right) \subseteq$ form $(G / P)=m(A)$. Если $A \notin K(G / P)$, то $b(A)=\varnothing \subseteq m(A)$. Поэтому $b \leq m$ и, значит, $\mathfrak{H} \subseteq \mathfrak{M}$.

Пусть $h$ - максимальньй внутренний $c$-экран формации $\mathfrak{H}$. Тогда по теореме 3.2 из [7] $h(A)=\mathfrak{H}$ и, значит, $m(A) \subseteq h(A)$. Поскольку $K(G) \backslash K(P)=K(G / P) \backslash K(P)$, то $m(C)=b(C) \subseteq h(C)$ для любого $C \in K(G) \backslash K(P)$. Следовательно, $m \leq h$. Тогда $\mathfrak{M}=C F(m) \subseteq C F(h)=\mathfrak{H}$. Из $\mathfrak{H} \subseteq M$ и $\mathfrak{M} \subseteq \mathfrak{H}$ следует, что $\mathfrak{H}=\mathfrak{M}$. Лемма доказана.

ЛЕмма 3. Пусть $G=[P] H-$ монолитическая группа с монолитом $P$, где $P$ әлементарная абелева $p$-группа, $H$ - монолитическая группа с неабелевым монолитом $R=H^{\mathfrak{N}}$. Если $\mathfrak{H}=c$ form $H-$ единственная максимальная с-подформация формаичи $\mathfrak{F}=c$ form $G$, mo $R=H^{\mathfrak{N}_{p}} \neq H$. 
ДокАЗАТЕЛЬСТво. Пусть $H$ - монолитическая группа с неабелевым монолитом $R$ $=H^{\mathfrak{N}}$ и $\mathfrak{H}=c$ form $H$. Тогда по следствию 52.34 из [12] $H-$ критическая, а значит, и формационно-критическая группа, причем ввиду леммы 18.2 из [11] $\mathfrak{M}=$ form $(H / R)-$ единственная максимальная подформация формации form $H$.

Пусть $G=[P] H$ - монолитическая группа с монолитом $P$, где $P \in \mathfrak{N}_{p}$. Так как $H-$ $f$-базисная группа, то по лемме 3.2 .2 из [10] $G$ - $c$-базисная группа, причем максимальная $c$-подформация $\mathfrak{L}$ из $\mathfrak{F}=c$ form $G$ обладает внутренним $c$-экраном $l$ со следующим строением:

$$
l(A)= \begin{cases}\mathfrak{M}, & A \in K(P), \\ \text { form } H & \forall A \in K(R), \\ (1) & \forall A \in K(G) \backslash(K(P) \cup K(P)), \\ \varnothing & \forall A \in \mathfrak{I} \backslash K(G) .\end{cases}
$$

Пусть $h$ - минимальньй $c$-экран формаций $\mathfrak{H}$. Предположим, что $K(P) \nsubseteq K(H)$. Рассмотрим групу $S=P \times H$. Так как $S / P \cong H \in \mathfrak{F}$ и $S / H \cong P \in \mathfrak{F}$, то $S \in \mathfrak{F}$. Поскольку $H \in c$ form $S$, то ввиду теоремы из [8] $\mathfrak{H} \subset \mathfrak{L}$. Пусть $s, f$ - минимальные $c$-экраны формаций $c$ form $S$ и $\mathfrak{F}$ соответственно. Тогда $s(A)=(1) \subset \mathfrak{H}=f(A)$ для $A \in K(P)$. Следовательно, $\mathfrak{L} \subset \mathfrak{F}$. Получили противоречие. Следовательно, $K(P) \subseteq K(H)$.

Так как $h(A)=l(A)$ для $A \in \mathfrak{I} \backslash K(P)$ и $(1)=h(A) \subset \mathfrak{M}=l(A)$ для $A \in K(P)$, то $\mathfrak{H} \subseteq \mathfrak{L}$. Пусть $\mathfrak{H}=\mathfrak{L}$.

Обозначим через $h_{1}, l_{1}$ максимальные внутренние $c$-экраны формаций $\mathfrak{H}, \mathfrak{L}$ соответственно. Ввиду [8, следствие 3] справедливо равенство $h_{1}=l_{1}$. Если $A \in K(P)$, то по теореме 3.2 из [7] и лемме 3.13 из [7] $h_{1}(A)=\mathfrak{N}_{p} h(A)=\mathfrak{N}_{p}(1)$. С другой стороны, $l_{1}(A)=\mathfrak{N}_{p} l(A)=\mathfrak{N}_{p} \mathfrak{M}$. Следовательно, $\mathfrak{N}_{p}(1)=\mathfrak{N}_{p} \mathfrak{M}$, что возможно только в том случае, когда $\mathfrak{M} \subseteq \mathfrak{N}_{p}$. Следовательно, $R=H^{\mathfrak{N}_{p}} \neq H$. Лемма доказана.

ОПРЕДЕЛЕНИЕ 13. Композиционная формация $\mathfrak{L}$ назьвается предмаксимальной $c$ подформацией $c$-формации $\mathfrak{F}$, если она является максимальной $c$-подформацией для некоторой максимальной $c$-подформации $c$-формации $\mathfrak{F}$.

ТЕОРема 1. Тогда и только тогда у неразрешимой с-формаиии $\mathfrak{F}$ все предмаксимальные с-подформации нильпотентны, когда $\mathfrak{F}=c$ form $G$, где группа $G$ удовлетворяет одному из следующих условий:

1) $G$ - монолитическая группа с неабелевым монолитом $P=G^{\mathfrak{N}}$;

2) $G=[M] H$ - монолитическая группа с монолитом $M$, где $M$ - точный

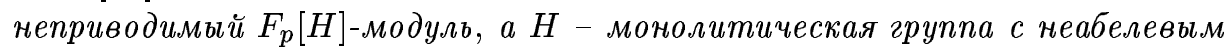
монолитом $R=H^{\mathfrak{N}_{p}} \neq H$;

3) $G$ - монолитическая группа с неабелевым монолитом $P$ и $c$ form $(G / P)$ $\mathfrak{N}_{c}$-критическая формачия;

4) $G=M \times H$, әде $M \cong Z_{p}$, а $H$ - монолитическая группа с неабелевым монолитом $R=H^{\mathfrak{N}}$ и $Z_{p} \notin K(H)$;

5) $G=M \times H$, где $H$ - монолитическая группа с неабелевым монолитом $R=H^{\mathfrak{N}}$, a $M-$ группа Шмидта, причем $K(M)=K(H / R) ;$

6) $G=M \times H$, где $M u H-$ не $f$-иниидентные монолитические группь $c$ неабелевыми монолитами $N=M^{\mathfrak{N}}$ и $R=H^{\mathfrak{N}}$ соответственно, причем $K(H / R)=K(M / N)$. 
ДокАЗАтЕльство. Необходимость. Так как $\mathfrak{F}$ - неразрешимая $c$-формация, то $\mathfrak{F}-$ ненильпотентная $c$-формация. Поскольку $\mathfrak{N}$ - формация классического типа, то по теореме 3.6.1 из [10] формация $\mathfrak{F}$ содержит по крайней мере одну $\mathfrak{N}_{c}$-критическую формацию $\mathfrak{H}$. Если $\mathfrak{F}=\mathfrak{H}$, то по теореме 1 из [13] $G$ - группа типа 1$)$ из условий теоремы.

Рассмотрим случай, когда $\mathfrak{H} \subset \mathfrak{F}$. В силу условий теоремы $\mathfrak{H}$ - максимальная $c$-подформация формации $\mathfrak{F}$.

Если $\mathfrak{F}$ содержит две неизоморфные простые групшы $A$ и $B$, хотя-бы одна из которых неабелева, то $A \times B \in \mathfrak{F}$ и, значит, $c \operatorname{form}(A \times B) \subseteq \mathfrak{F}$. Пусть $A \notin \mathfrak{A}$. Так как $c$ form $A \subset c$ form $(A \times B)$, то $\mathfrak{F}=c$ form $(A \times B)$. Если $B \in \mathfrak{A}$, то групша $G=A \times B-$ группа типа 4) из условий теоремы. Если $B \notin \mathfrak{A}$, то $G=A \times B$ - группа типа 6) из условий теоремы.

Пусть $C \in \mathfrak{F}$ и $C$-простая неабелева группа. Ввиду вышесказанного можем считать, что каждая простая группа из $\mathfrak{F}$ изоморфна $C$. Так как по теореме 1 из [13] $c$ form $C-$

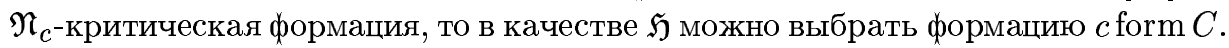

Пусть $G \in \mathfrak{F} \backslash \mathfrak{H}$ и $G$ - группа наименьшего порядка с таким свойством. Тогда $G-$ монолитическая группа с монолитом $P=G^{\mathfrak{H}}$ и $\mathfrak{F}=c$ form $G$. Допустим, что $P$ - элементарная абелева $p$-группа. Тогда из теоремы в [8] следует, что $Z_{p} \in \mathfrak{F}$ и, значит, $Z_{p} \cong C$. Получили противоречие. Следовательно, монолит $P$ неабелев. Допустим, что $G=P$. Тогда $P$ - простая группа из $\mathfrak{F}$ и, значит, $P \cong C$. Но тогда $G \in \mathfrak{H}$. Получили противоречие. Следовательно, $P \subset G$ и $G$ - группа типа 3$)$ из условий теоремы.

Пусть $\mathfrak{F}$ не содержит неабелевых простьх групп.

Рассмотрим случай, когда $\mathfrak{H}$ - единственная максимальная $c$-подформация формации $\mathfrak{F}$. Выберем группу $G \in \mathfrak{F} \backslash \mathfrak{H}$, причем минимального порядка, с таким свойством. Тогда $G$ - монолитическая групша с монолитом $P=G^{\mathfrak{H}}$ и $\mathfrak{F}=c$ form $G$.

Пусть $P$ - элементарная абелева $p$-группа. Поскольку $G \notin \mathfrak{S}$, то и $G / P \notin \mathfrak{S}$. Следовательно, $c$ form $(G / P)=\mathfrak{H}$. Согласно теореме 1 из [13] $\mathfrak{H}=c$ form $H$, где $H$ - монолитическая группа с неабелевым монолитом $R=H^{\mathfrak{N}}$ и по теореме из [8] минимальньй $c$-экран $h$ формации $\mathfrak{H}$ имеет следующее строение:

$$
h(A)= \begin{cases}\text { form } H, & A \in K(R), \\ (1) & \forall A \in K(H) \backslash K(R), \\ \varnothing & \forall A \in \mathfrak{I} \backslash K(H) .\end{cases}
$$

Допустим, что главньй фактор $P / 1 \cong P$ централен в группе $G$. Как и при доказательстве леммы 3 , нетруднопоказать, что $K(P) \subseteq K(G / P)$ и, значит, $K(G)=K(G / P)$. Тогда по лемме $1 c$ form $G=c \operatorname{form}(G / P) \subseteq \mathfrak{H}$. Получили противоречие. Следовательно, $P$ не является центральньп главным фактором групшы $G$.

Рассмотрим группу $S=[P](G / P)$. По лемме 3.32 из [11] $S \in$ form $G \subseteq c$ form $G$, причем $|G|=|S|$. Предположим, что $c$ form $S \subset c$ form $G$. Тогда $c$ form $S \subseteq \mathfrak{H}$. Пусть $s$ - минимальный $c$-экран формации $c$ form $S$. Так как $C_{S}(P) \subset S$, то по теореме из [8] $h(A)=(1) \subset s(A)$ для $A \in K(P)$. Но по [8, следствие 2] $s(A) \subseteq h(A)$ для $A \in \mathfrak{I}$. Получили противоречие. Следовательно, $\mathfrak{F}=c$ form $S$ и $c$ form $H$ - неразрешимая $\mathfrak{N}_{c^{-к р и т и-~}}$ ческая формация, причем $Z_{p} \in K(H)$.

Поскольку $\mathfrak{H}=c$ form $(G / P)$, то по теореме из [8]

$$
h(A)=\text { form } H \subseteq \text { form }\left((G / P) / F_{A}(G / P) \subseteq \text { form }(G / P)\right)
$$


для $A \in K(R)$. Следовательно, $H \in \operatorname{form}(G / P)$. Поскольку $O_{p}(H)=1$, то по [11, лемма 18.8] существует точньй неприводимьй $F_{p}[H]$-модуль $M$, где $F_{p}$ - поле из $p$ элементов.

Рассмотрим групшу $B=[M] H$. Пусть $\mathfrak{B}=c$ form $B$ и $b$ - минимальный $c$-экран формации $\mathfrak{B}$. Тогда по теореме из [8]

$$
b(A)= \begin{cases}\text { form } H, & A \in K(P), \\ \text { form } H, & A \in K(R), \\ (1) & \forall A \in K(\mathfrak{B}) \backslash(K(R) \cup K(P)), \\ \varnothing & \forall A \in \mathfrak{I} \backslash K(\mathfrak{B}) .\end{cases}
$$

Поскольку $G$ - монолитическая группа с монолитом $P=G^{\mathfrak{N}}$ и $K(\mathfrak{F})=K(\mathfrak{B})$, то по теореме из [8] минимальньй $c$-экран $f$ формации $\mathfrak{F}$ имеет следующее строение:

$$
f(A)= \begin{cases}\text { form }(G / P), & A \in K(P), \\ \text { form } H, & A \in K(R), \\ (1) & \forall A \in K(\mathfrak{B}) \backslash(K(R) \cup K(P)), \\ \varnothing & \forall A \in \mathfrak{I} \backslash K(\mathfrak{B}) .\end{cases}
$$

Так как form $H \subseteq$ form $(G / P)$, то $b(A) \subseteq f(A)$ для $A \in \mathfrak{I}$. Следовательно, $\mathfrak{B} \subseteq \mathfrak{F}$. Так как $\mathfrak{H} \subset \mathfrak{B}$, то $\mathfrak{F}=\mathfrak{B}$ и по лемме $3 R=H^{\mathfrak{N}_{p}} \neq H$. Полагая $G=B$ получим, что $G$ удовлетворяет условию 2) теоремы.

Пусть $P$ - неабелевая групш; тогда $G$ - группа типа 3$)$ из условий теоремы.

Рассмотрим случай, когда $\mathfrak{F}$ содержит различные максимальные $c$-подформации $\mathfrak{M}$ и $\mathfrak{H}$. Тогда $\mathfrak{F}=c \operatorname{form}(\mathfrak{H} \cup \mathfrak{M})$ и по теореме из $[8] K(\mathfrak{F})=K(\mathfrak{H}) \cup K(\mathfrak{M})$. Так как $\mathfrak{F}$ - неразрешимая формация, хотя бы одна из формаций $\mathfrak{H}$ или $\mathfrak{M}$ неразрешима. Пусть

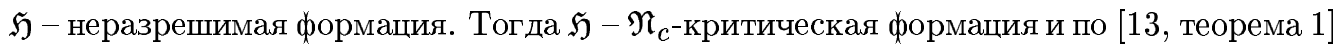
$\mathfrak{H}=c$ form $H$, где $H$ - монолитическая группа с неабелевым монолитом $R=H^{\mathfrak{N}}$. Пусть $D \in K(\mathfrak{F}) \backslash K(\mathfrak{H})$ и $D$ - абелева групша. Тогда $D \in \mathfrak{F}$ и $D \times H \in \mathfrak{F}$. Так как $\mathfrak{H} \subset c$ form $(D \times H)$, то $\mathfrak{F}=c$ form $(D \times H)$ и, значит, $G=D \times H-$ группа типа 4) из условий теоремы. Поэтому в дальнейшем можем считать, что каждая абелева группа из $K(\mathfrak{F})$ принадлежит $\mathfrak{H}$, а значит, каждая нильпотентная группа из $\mathfrak{F}$ принадлежит $\mathfrak{H}$. Так как $\mathfrak{M}$ не является $c$-подформацией формации $\mathfrak{H}$, то $\mathfrak{M}$ - ненильпотентная формация.

Если $\mathfrak{M}$ - разрешимая формация, то по [13, теорема 1] $\mathfrak{M}=c$ form $M$, где $M$ - группа Шмидта. Тогда $M \times H \in \mathfrak{F}$ и, значит, $\mathfrak{F}=c$ form $(M \times H)$, причем $K(M) \subseteq K(H) \backslash K(R)$. Предположим, что $K(H) \backslash K(R) \nsubseteq K(M)$. Тогда возьмем $L \in(K(H) \backslash K(R)) \backslash K(M)$, где $L$ - элементарная абелева $p$-группа. Так как $L \in \mathfrak{F}$, то $L \times M \in \mathfrak{F}$ и, значит, $\mathfrak{F}=c$ form $(L \times M) \subseteq \mathfrak{S}$. Получили противоречие. Следовательно, $K(H) \backslash K(R) \subseteq K(M)$. Таким образом, $K(M)=K(H) \backslash K(R)$ и, значит, $G=M \times H-$ группа типа 5) из условий теоремы.

Если $\mathfrak{M}$ - неразрешимая формация, то по [13, теорема 1] $\mathfrak{M}=c$ form $M$, где $M-$ монолитическая група с неабелевым монолитом $N=M^{\mathfrak{N}}$. Тогда $M \notin$ form $H$ и $H \notin$ form $M$. Следовательно, $M$ и $H$ не являются $f$-инцидентньми группами.

Рассмотрим группу $G=H \times M \in \mathfrak{F}$. Тогда $\mathfrak{H} \subset c$ form $G$ и, значит, $\mathfrak{F}=c$ form $G$. 
Предположим, что $K(H / R) \neq K(M / N)$ и $A \in K(H / R) \backslash K(M / N)$. Тогда $A \notin M$ и, значит, $\mathfrak{F}=c \operatorname{form}(A \times M)$ и по теореме из [8] $K(\mathfrak{F})=K(A) \cup K(M)=K(H) \cup K(M)$. Пусть $K(R)=K(N)=(C)$, где $C$ - простая неабелева группа. Обозначим через $f$ минимальньй $c$-экран формации $\mathfrak{F}$. Тогда из $\mathfrak{F}=c$ form $(A \times M)$ следует, что $f(C)=$ form $M$. $\mathrm{C}$ другой стороны, из $\mathfrak{F}=c$ form $(H \times M)$ следует, что $f(C)=$ form $(H \times M) \subset$ form $M=$ $f(C)$. Получили противоречие. Следовательно, $K(H / R)=K(M / N)$ и $G$-группа типа 6) из условия теоремы.

Достаточность. Пусть $\mathfrak{F}=c$ form $G$. Если $G$ - монолитическая группа с неабелевым монолитом $P=G^{\mathfrak{N}}$, то по [13, теорема 1] $\mathfrak{F}-\mathfrak{N}_{c}$-критическая формация. Следовательно, все предмаксимальные $c$-подформации из формации $\mathfrak{F}$ нильпотентны.

Если $G=[M] H-$ монолитическая группа с монолитом $M$, где $M$ - точньй неприводимьй $F_{p}[H]$-модуль, а $H$ - монолитическая группа с неабелевым монолитом $R=$ $H^{\mathfrak{N}_{p}} \neq H$, то $H-f$-базисная групша, причем $\mathfrak{M}=$ form $(H / R)$ - максимальная подформация формации form $H$ и по [10, лемма 3.2.2] $G-c$-базисная группа, и формация $\mathfrak{F}=c$ form $G$ содержит единственную максимальную $c$-подформацию $\mathfrak{L}$ с внутренним $c$-экраном $l$, имеющим следующее строение:

$$
l(A)= \begin{cases}\mathfrak{M}, & A \in K(P), \\ \text { form } H, & A \in K(R), \\ (1) & \forall A \in K(G) \backslash(K(P) \cup K(R)), \\ \varnothing & \forall A \in \mathfrak{I} \backslash K(G) .\end{cases}
$$

Пусть $\mathfrak{H}=c$ form $H$. По теореме из $[8] \mathfrak{H} \subset \mathfrak{F}$ и, значит, $\mathfrak{H} \subseteq \mathfrak{L}$. Предположим, что формация $\mathfrak{L} \nsubseteq \mathfrak{H}$.

Выберем группу $L \in \mathfrak{L} \backslash \mathfrak{H}$, причем наименьшего порядка с таким свойством. Тогда $L$ - монолитическая группа с монолитом $N=L^{\mathfrak{H}}$ и $\mathfrak{L}=c$ form $L$. Пусть $m$ - минимальный $c$-экран формации $\mathfrak{L} ;$ тогда $m \leq l$.

Если $N$ - элементарная абелева $q$-группа и $l_{1}$ - максимальньй внутренний $c$-экран формации $\mathfrak{L}$, то $l_{1}(A)=\mathfrak{N}_{q} l(A)=\mathfrak{N}_{q}$ для $A \in K(N) ;$ с другой стороны, $l_{1}(A)=$ $\mathfrak{N}_{q} m(A)=\mathfrak{N}_{q}$ form $(L / N)$. Следовательно, form $(L / N) \subseteq \mathfrak{N}_{q}$. Поскольку $\mathfrak{L} \nsubseteq \mathfrak{S}$ и $N \in \mathfrak{A}$, то $L / N \notin \mathfrak{S}$. Получили противоречие.

Пусть $N$ - неабелев монолит. Тогда $m(A)=$ form $L \subseteq l(A)=$ form $H \subseteq c$ form $H=\mathfrak{H}$ для $A \in K(N)$ и, значит, $L \in \mathfrak{H}$. Получили противоречие. Следовательно, $\mathfrak{H}=\mathfrak{L}$.

Так как по $[13$, теорема 1$] \mathfrak{H}-\mathfrak{N}_{c}$-критическая $c$-формация, то все предмаксимальные $c$-подформации из $\mathfrak{F}$ нильпотентны.

Если $G$-монолитическая группа с неабелевым монолитом $P$ и $c$ form $(G / P)-\mathfrak{N}_{c}$-критическая формация, то по лемме $2 \mathfrak{F}$ содержит единственную максимальную $c$-подформацию $c$ form $(G / P)$. Поскольку $c$ form $(G / P)-\mathfrak{N}_{c}$-критическая формация, то все предмаксимальные $c$-подформации формации $\mathfrak{F}$ нильпотентны.

Пусть $G=M \times H$, где $M \cong Z_{p}, H$ - монолитическая группа с неабелевым монолитом $R=H^{\mathfrak{N}}$ и $Z_{p} \notin K(H)$. Тогда $\mathfrak{F}=c$ form $(M \times H)=c$ form $M \vee^{c} c$ form $H$, и по лемме 8 из [13] каждая ненильпотентная $c$-подформация формации $\mathfrak{F}$ содержит формацию $c$ form $H$. Но такими формациями являются только $\mathfrak{F}$ и $c$ form $H$. Следовательно, $c$ form $H$ - максимальная $c$-подформация формации $\mathfrak{F}$ и, значит, все собственные $c$-подформации из $\mathfrak{F}$, отличные от $c$ form $H$, нильпотентны. Следовательно, все предмаксимальные $c$-подформации из $\mathfrak{F}$ нильпотентны. 
Пусть $G=M \times H$, где $M=M_{p}\left[M_{q}\right]$ - групша Шмидта, $H$ - монолитическая группа с неабелевым монолитом $R=H^{\mathfrak{N}}$, причем $K(M)=K(H / R)$. Пусть $f, m, h-$ минимальные $c$-экраны формаций $\mathfrak{F}, c$ form $M, c$ form $H=\mathfrak{H}$ соответственно. Тогда в силу теоремы из [8] $f\left(Z_{q}\right)=$ form $Z_{p}, h\left(Z_{q}\right)=(1)$ и $f(A)=h(A)$ для любого А $\in \mathfrak{I} \backslash\left(Z_{q}\right)$. Из строения экранов $f$ и $h$ следует, что формация $c$ form $H$ - максимальная $c$-подформация формации $\mathfrak{F}$. Покажем,что $c$-формация $c$ form $M$ - максимальная $c$-подформация формации $\mathfrak{F}$. Пусть формация $\mathfrak{L} \subset \mathfrak{F}$. Предположим, что $c$ form $M \subset \mathfrak{L}$. Обозначим через $l$ минимальный $c$-экран формации $\mathfrak{L}$. Поскольку $f(A)=m(A)$ для $A \in K(M)$, то $K(\mathfrak{L})=K(\mathfrak{F})$. Так как $\mathfrak{L} \subset \mathfrak{F}$, то $l(A) \subset f(A)$ для $A \in K(R)$. Но $f(A)=$ form $H$ для $A \in K(R)$. В силу [11, лемма 18.2] $l(A) \subseteq \operatorname{form}(H / R) \subset \mathfrak{N}$. Тогда по лемме 2 из [13] $l(A)=\varnothing$. Получили противоречие. Следовательно, $\mathfrak{L}=c$ form $M$ - максимальная $c$-подформация формации $\mathfrak{F}$.

Из строения $c$-экранов $l, m$ и $f$ следует, что максимальных $c$-подформаций формации $\mathfrak{F}$, отличных от $\mathfrak{H}$ и $\mathfrak{L}$, нет. Поскольку по $[13$, теорема 1$]$ формации $\mathfrak{H}$ и $\mathfrak{M}-\mathfrak{N}_{c}$-критические, все предмаксимальные $c$-подформации формации $\mathfrak{F}$ нильпотентны.

Если $G=M \times H$, где $M$ и $H$ - не $f$-инцидентные монолитические группы с неабелевыми монолитами $N=M^{\mathfrak{N}}$ и $R=H^{\mathfrak{N}}$ соответственно, причем $K(H / R)=K(M / N)$, то $\mathfrak{F}=c$ form $H \vee^{c} c$ form $M$. Пусть $f$ - минимальньй $c$-экран формации $\mathfrak{F}$. Тогда если $K(R) \neq K(N)$, то по теореме из [8]

$$
f(A)= \begin{cases}\operatorname{form} H, & A \in K(R), \\ \operatorname{form} M, & A \in K(N), \\ (1) & \forall A \in K(\mathfrak{F}) \backslash(K(R) \cup K(N)), \\ \varnothing & \forall A \in \mathfrak{I} \backslash K(\mathfrak{F}) .\end{cases}
$$

Покажем, что формация $\mathfrak{H}=c$ form $H$ - максимальная $c$-подформация формации $\mathfrak{F}$. Так как по теореме из [8] для минимального $c$-экрана $h$ формации $\mathfrak{H} h(A)=f(A)$ для $A \in K(H)$, существует $c$-формация $\mathfrak{L} \subset \mathfrak{F}$ такая, что для минимального $c$-экрана $l$ формации $\mathfrak{L}$ справедливо $l(A)=h(A)=f(A)$ для $A \in K(H)$ и $\varnothing=h(A) \subset l(A)$ для $A \in K(L) \backslash K(H)$. Из строения $c$-экрана $f$ следует, что $K(\mathfrak{L}) \backslash K(H)=K(N)$. Пусть $K(N)=(D)$; тогда $l(D) \subset f(D)=$ form $M$. По лемме 18.2 из [11] $l(D) \subseteq$ form $(M / N)$ $\subset \mathfrak{N}$. Тогда по лемме 2 из [14] $l(D)=\varnothing$. Таким образом, получили, что $l=h$. Следовательно, $\mathfrak{H}$ - максимальная $c$-подформация формации $\mathfrak{F}$. Аналогично можно показать, что формация $\mathfrak{M}=c$ form $M$ - максимальная $c$-подформация формации $\mathfrak{F}$.

Пусть $\mathfrak{B}$ - собственная произвольная ненильпотентная $c$-подформация формации $\mathfrak{F}$ и $b$ - минимальньй $c$-экран формации $\mathfrak{B}$. Тогда $K(R) \subseteq K(\mathfrak{B})$ или $K(N) \subseteq K(\mathfrak{B})$. Но, как показано вьше, $b(A)=$ form $H$ для $A \in K(R)$ и $b(A)=$ form $M$ для $A \in K(N)$. Таким образом, $\mathfrak{L}$ может совпадать только с формациями $\mathfrak{H}$ или $\mathfrak{M}$. Следовательно, все предмаксимальные $c$-подформации формации $\mathfrak{F}$ нильпотентны.

Пусть $K(R)=K(N)$, тогда по теореме из [8]

$$
f(A)= \begin{cases}\text { form } G, & A \in K(R), \\ (1) & \forall A \in K(G) \backslash K(R), \\ \varnothing & \forall A \in \mathfrak{I} \backslash K(G) .\end{cases}
$$


Но по [11, лемма 3.37] все ненильпотентные критические групшы из form $G$ являются гомоморфными образами группы $G$. Следовательно, ненильпотентными подформациями в $\mathfrak{F}$ будут только формации $c$ form $(G / R)$ и $c$ form $(G / N)$. Таким образом, все предмаксимальные $c$-подформации из $\mathfrak{F}$ нильпотентны. Теорема доказана.

ЗАмЕчАниЕ. В работе [5] были описаны локальные формации с предмаксимальными локальными подформациями. Поскольку каждая разрешимая $c$-формация является локальной формацией, то полностью описаны все $c$-формации с предмаксимальными нильпотентньгми $c$-подформациями.

Автор выражает благодарность профессору В.А. Ведерникову за ценные советы и внимание к данной работе.

\section{СПИСОК ЦИТИРОВАННОЙ ЛИТЕРАТУРЫ}

[1] Birkhoff G. On structure of algebras // Proc. Carbridge Phill. Soc. 1935. V. 31. P. 433-454.

[2] Neumann B. N. Indentical relation in groups. I // Math. Ann. 1937. V. 114. P. 506-525.

[3] Gaschutz W. Zur Teorie der endlichen auflosbaren Gruppen // Math. Z. 1963. V. 80. № 4. P. 300-305.

[4] Скиба А.Н. О критических формациях // Весці АН БССР. Сер. фіз-мат. наук. 1980. № 4. C. $27-33$.

[5] Таргонский Е. А. О локальных формациях с нильпотентньми немаксимальными собственными локальными подформациями. Вопросы алгебры. 1-й выпуск. Минск: Изд-во Университетское, 1985.

[6] Шеметков Л.А. Два направления развития теории непростых конечных групп // УМН. 1975. Т. 30. № 2. C. 179-198.

[7] Шеметков Л. А. Формации конечных групп. М.: Наука, 1978.

[8] Скиба А. Н., Шеметков Л. А. О минимальном композиционном экране композиционной формации // Вопросы алгебры.. Гомель: Изд-во Гомельского ун-та. Вып. 7. С. 39-43.

[9] Ведерников В.А., Сорокина М. М. Композиционные наследственные критические формации // Вопросы алгебры. Гомель: Изд-во Гомельского ун-та. Вып. 11. С. 6-19.

[10] Сорокина М. М. Композиционные критические формации. Дисс. .. к. ф..-м.н. Брянск, 1998.

[11] Шеметков Л. А., Скиба А. Н. Формации алгебраических систем. М.: Наука, 1989.

[12] Нейман Х. Многообразия групп. М.: Мир, 1969.

[13] Чиспияков С. В. О композиционных формациях с заданными системами нильпотентных подформаций. Брянск: Брянский госпедуниверситет, 1998. (Рукопись депонирована в ВИНИТИ 26 октября 1998 г., № 3098-В98).

[14] Коптюх Д. Г. Композиционные формации $c$-длины 2. Брянск: Брянский госпедуниверситет, 1998. (Рукопись депонирована в ВИНИТИ 26 октября 1998 г., № 3099-В98).

Брянский государственный педагогический университет им. И. Г. Петровского П Поступило 